\title{
Intravesical therapy options for neurogenic detrusor overactivity
}

\author{
André Reitz*,1 and Brigitte Schurch ${ }^{1}$ \\ ${ }^{1}$ Neuro-Urology, Spinal Cord Injury Center, Balgrist University Hospital, Zurich, Switzerland
}

Study design: Review article.

Setting: Neuro-Urology, Spinal Cord Injury Center, Balgrist University Hospital, Zurich, Switzerland.

Objectives: This review considers intravesical treatment options of neurogenic detrusor overactivity and discusses the underlying mechanism of action, clinical safety and efficacy, and the future trends.

Methods: The available literature was reviewed using medline services.

Results: Oral anticholinergic drugs are widely used to treat detrusor overactivity, but they are ineffective in some patients or cause systemic side effects such as blurred vision or dry mouth. As an alternative, topical therapy strategies have been suggested to achieve a profound inhibition of the overactive detrusor and to avoid high systemic drug levels. Currently available intravesical treatment options either act on the afferent arc of the reflex such as local anaesthetics or vanilloids or on the efferent cholinergic transmission to the detrusor muscle such as intravesical oxybutynin or botulinum toxin. Although an established and effective therapy, intravesical oxybutynin is not widely used. Evidence for clinical significance of intravesical atropine and local anaesthetic is missing. Intravesical capsaicin has been shown to improve clinical and urodynamic parameters, but cause pain in some patients. The intravesical instillation of resiniferatoxin and the injection of botulinum-A toxin into the detrusor muscle are promising new options; however, randomised placebo-controlled studies to prove their safety and efficacy are still missing.

Conclusion: Intravesical treatment strategies in patients with neurogenic detrusor overactivity may provide alternatives to established therapies such as oral anticholinergics. The selectivity of the intravesical treatment and the reduction or even the absence of side effects are major advantages of this topical approach.

Spinal Cord (2004) 42, 267-272. doi:10.1038/sj.sc.3101584; Published online 3 February 2004

Keywords: bladder; neurogenic; administration; intravesical; anticholinergic agents; capsaicin; botulinum toxins

\section{Introduction}

Urinary incontinence due to detrusor overactivity remains an enormous problem for people with neurological disorders. According to the standardisation of terminology of lower urinary tract function published by the International Continence Society, detrusor overactivity is a urodynamic observation characterised by involuntary detrusor contractions during the filling phase which may be spontaneous or provoked. According to the cause this observation may be qualified to neurogenic detrusor overactivity when there is a relevant neurologic condition. ${ }^{1}$

*Correspondence: A Reitz, Neuro-Urology, Spinal Cord Injury Center, Balgrist University Hospital, Forchstrasse 340, 8008 Zurich, Switzerland
A considerable number of disturbances of the lower urinary tract are caused by neurological disorders such as spinal cord injury, multiple sclerosis, meningomyelocele and other diseases affecting brain structures or spinal pathways involved in the control of bladder and urethra. Many patients suffer from urinary incontinence and the urodynamic examination usually reveals a neurogenic detrusor overactivity. This pathology is based on a spinal reflex arc involving small unmyelinated type $\mathrm{C}$ fibres that become relevant after disconnection of the bladder from higher centres. ${ }^{2}$ Treatment options either act on the afferent arc of the reflex such as local anaesthetics or vanilloids or on the efferent cholinergic transmission to the detrusor muscle such as oral anticholinergic drugs (Figure 1). These agents are widely used to treat detrusor overactivity. However, 


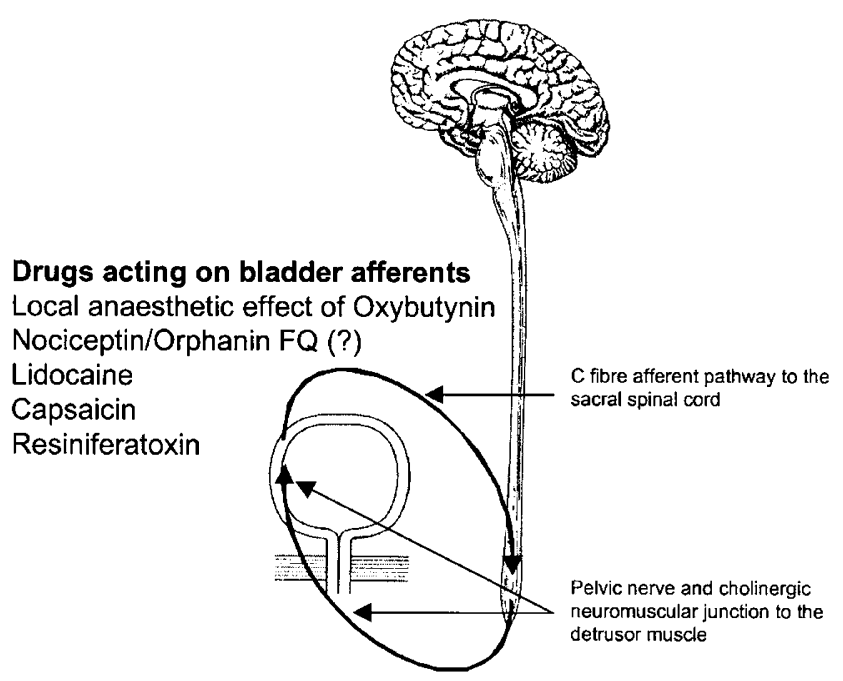

Drugs acting the efferent cholinergic transmission Atropine

Anticholinergic effect of Oxybutynin

Botulinum-A toxin

Figure 1 Targets for intravesical therapy options to treat neurogenic detrusor overactivity - blocking afferent $\mathrm{C}$ fibres or efferent cholinergic transmission

they are ineffective in some patients or cause systemic side effects. Anticholinergic side effects such as blurred vision or dry mouth limit the applicable dose.

As an alternative, topical therapy strategies have been suggested to achieve a profound inhibition of the overactive detrusor and to avoid high systemic drug levels. This review considers the available literature concerning the intravesical treatment options of neurogenic detrusor overactivity and discusses the underlying mechanism of action, clinical safety and efficacy, and the future trends.

\section{Intravesical local anaesthetics}

In clinical practice, intravesical local anaesthetics have been used for diagnostic and therapeutic purpose. Intravesical administration of lidocaine produces effective mucosal anaesthesia and was found to increase bladder capacity. ${ }^{3,4}$ In children with meningomyelocele intravesical lidocaine could improve bladder capacity and compliance and decrease the number of uninhibited detrusor contractions. ${ }^{5}$ Also, intravesical bupivacaine was found to have an effect in patients with spinal cord injury. ${ }^{6}$ However, the effect of both drugs is only of short duration and long-term observations are not available. There is some evidence that intravesical lidocaine could be used to differentiate between neurogenic and idiopathic detrusor overactivity ${ }^{7}$ and within the group of neurological disturbed patients to distinguished between detrusor overactivity caused by spinal cord or brain lesions. ${ }^{8}$ Some results suggest that the instillation of lidocaine could predict which patients might respond to intravesical vanilloids. ${ }^{8}$ However, in another study the procedure was not reliable to predict the clinical response to oral oxybutynin. ${ }^{5}$

\section{Intravesical vanilloids}

Capsaicin and resiniferatoxin are vanilloids that act on bladder afferents transmitting sensory information from urothelium and bladder muscle layers to the spinal cord and brain. When applied into the bladder, vanilloids activate nociceptive type $\mathrm{C}$ fibres that convey noxious signals and induce painful sensations. Both substances bind a nonselective cation channel known as vanilloid receptors type 1 . These receptors have been demonstrated in rat by immunohistochemistry in urothelium, basal lamina and smooth muscle layers. ${ }^{9}$ Also, in the human bladder this receptor has been identified recently and vanilloid receptors type 1 immunoreactive fibers were found to be scattered throughout the suburothelium and traverse muscle layers. ${ }^{10}$ Binding of vanilloids to the vanilloid receptors type 1 initially induces excitement and later desensitisation of the $\mathrm{C}$ fibres. Pathological sensitisation or recruitment of these fibres, for example, following spinal cord injury are involved in inducing bladder overactivity. Vanilloids block these afferent fibres and interrupt the pathological reflex activity.

\section{Intravesical capsaicin}

The first study in 1991 by Fowler et al ${ }^{11}$ demonstrated in patients with neurogenic bladder due to multiple sclerosis that intravesical instillation of $100 \mathrm{ml}$ of $1-2$ $\mathrm{mmol} / \mathrm{l}$ capsaicin in $30 \%$ ethanol in saline was effective in reducing neurogenic detrusor overactivity. Since this first report at least 12 studies were published but only two were randomised placebo-controlled trials. ${ }^{12,13}$ Both studies tested the effects of $30 \mathrm{mg}$ capsaicin in $30 \%$ ethanol versus ethanol alone and found that intravesical capsaicin significantly improved clinical and urodynamic parameters in patients with neurogenic detrusor overactivity. In contrast, another placebocontrolled crossover study could not find $1 \mathrm{mmol}$ capsaicin superior to saline. ${ }^{14}$ In a meta-analysis, the results of 115 patients treated in seven centres were analysed. ${ }^{15}$ In this population, $97(84.3 \%)$ were found to have at least partial remission of symptoms. In addition, the urodynamic parameters improved, the mean increase of the maximum cystometric bladder capacity varied from 53 to $151 \mathrm{ml}$ and the mean decrease of detrusor pressure from 17 to $30 \mathrm{~cm} \mathrm{H}_{2} \mathrm{O}$. The preinstillation of lidocaine reduced pain and did not influence mechanism of action of capsaicin. ${ }^{16}$ The duration of effect of a single instillation of capsaicin may exceed 6 month $^{15,17}$ and repeated instillations resulted in the same benefit and duration of the effect. ${ }^{18,19}$ 


\section{Intravesical resiniferatoxin}

Resiniferatoxin, an ultrapotent capsaicin analogue, acts also on the vanilloid receptor and induces, as in capsaicin, a desensitisation on the afferent bladder innervation. ${ }^{20}$ Compared to capsaicin, the major advantage is that resiniferatoxin acts without the initial excitatory effect on the nerve fibres which may be responsible for pain observed after capsaicin instillation. The initial study by Cruz et $a l^{21}$ demonstrated, in patients with neurogenic detrusor overactivity, that intravesical instillation of resiniferatoxin $(50-100 \mathrm{nmol} /$ 1) could improve clinical as well as urodynamic parameters for a period of up to 3 month. Being more potent, resiniferatoxin seems to be also more efficient than capsaicin. ${ }^{22,23}$ Patients who failed to respond to capsaicin were successfully treated with resiniferatoxin. ${ }^{23}$ Silva et $a l^{24}$ reported that the effect of a single instillation of $50-100 \mathrm{nM}$ resiniferatoxin lasted up to 12 months in seven out of 13 patients.

Two randomised studies are currently available, one comparing resiniferatoxin to placebo in patients with hypersensitive disorder or bladder pain and the other comparing resiniferatoxin to capsaicin in patients with neurogenic detrusor overactivity. Resiniferatoxin $(10 \mathrm{nM})$ was found to be superior than placebo in non-neurogenic patients. ${ }^{25}$ Secondly, a recent prospective randomised study comparing the two vanilloids ( $2 \mathrm{mM}$ capsaicin versus $100 \mathrm{nM}$ resiniferatoxin) demonstrated that the intravesical administration of resiniferatoxin is superior to that of capsaicin for both clinical benefit and improvement of urodynamic parameters. ${ }^{22}$ Randomised and placebo-controlled trials of intravesical resiniferatoxin in neurogenic patients are still not available.

\section{Intravesical nociceptin/orphanin FQ}

Nociceptin/orphanin FQ is a neuropeptide that influence physiological processes by activating a specific $G$ protein-coupled receptor called opioid receptor-like OP4. ${ }^{26,27}$ In rats, this peptide has been reported to inhibit the voiding reflex. ${ }^{28} \mathrm{~A}$ study in humans investigated the effect of nociceptin/orphanin FQ in healthy subjects and patients with neurogenic detrusor overactivity. ${ }^{29}$ After intravesical instillation of $1 \mu \mathrm{M}$ nociceptin/orphanin FQ, a significant increase in mean bladder cystometric capacity and volume threshold for the appearance of neurogenic detrusor overactivity in patients compared to healthy subjects group was observed. However, the clinical and urodynamic effect lasted for 2 weeks only. In this study, it was also hypothetised that nociceptin/orphanin FQ acts on the afferent limb of the micturition reflex in spinal cord injured patients and activates inhibitory opoid receptorlike $\mathrm{OP}_{4}$ expressed on afferent type $\mathrm{C}$ fibres of the bladder. ${ }^{29}$ Recently, a randomised, placebo-controlled and double-blind study on 14 patients with neurogenic detrusor overactivity has been published and the results indicated that $1 \mu \mathrm{M}$ nociceptin/orphanin $\mathrm{FQ}$ provides an acute inhibitory effect on the micturition reflex in this patient population. ${ }^{30}$ The bladder capacity and the threshold for the appearance of detrusor overactivity increased significantly from 139 to $240 \mathrm{ml}$ and from 84 to $201 \mathrm{ml}$, respectively. Since this was an acute experiment, the duration of the effect as well as the ideal dose remain still unknown.

\section{Intravesical atropine}

When applied intravesically the unselective antimuscarinic agent atropine acts on the efferent arc of bladder innervation and blocks the cholinergic transmission to the detrusor muscle. The intravesical administration of the drug has been studied in patients with neurogenic detrusor overactivity due to spinal cord injury and multiple sclerosis. In spinal cord injury, atropine could reduce the maximum detrusor pressure during an uninhibited contraction and increase the reflex volume as well as the volume when the first leak occurred. ${ }^{31} \mathrm{~A}$ significant increase in bladder capacity was demonstrated in a placebo-controlled crossover study in patients with multiple sclerosis. ${ }^{32}$ The systemic antimuscarinic activity was studied recently and intravesical instillation of atropine was not found to have any systemic antimuscarinic side effects. ${ }^{33}$ The duration of the effect has not been exactly determined, but it was suspected to last less than $6 \mathrm{~h}$.

\section{Intravesical oxybutynin}

Oxybutynin chloride is a tertiary amine with antimuscarinic, spasmolytic, and local anaesthetic properties. The intravesical instillation of oxybutynin had been suggested in the late eighties instead of oral administration of the drug to avoid high plasma levels and systemic side effects. ${ }^{34}$ When comparing the oral to the intravesical administration, the instillation provides a profound reduction of occurrence and severity of side effects. ${ }^{34-36}$ However, after intravesical instillation there is a substantial absorption of the drug. Pharmacokinetic studies indicated that the effects on the detrusor muscle are systemic due to its absorption on the blood. ${ }^{36,37}$ Recent findings revealed that serum levels after intravesical instillation are as high as after oral intake, ${ }^{38}$ suggesting another mechanism for less side effects after instillation. Different ratios between the drug and its metabolites as well as a reduced first pass mechanism after instillation have been discussed. ${ }^{39}$

Target structures of the intravesical oxybutynin are the bladder afferents by a local anaesthetic effect on type C fibres ${ }^{40}$ and the efferent cholinergic transmission by an anticholinergic effect. Several reports have been published over the years that established safety and efficacy of intravesical oxybutynin in adults ${ }^{34-36,41}$ and children $^{42-45}$ with neurogenic detrusor overactivity. A dual therapy strategy combining oral and intravesical oxybutynin ( $15 \mathrm{mg}$ three times daily) was found to be more effective than oral treatment alone. ${ }^{46}$ However, there is only one randomised and placebo-controlled study in a 
small population reporting a clinical benefit in patients with neurogenic and idiopathic detrusor overactivity. ${ }^{38}$

Although an effective treatment, current problems limiting the clinical use are the necessity of catheterisation and the high costs of special preparations due to lack of commercially available solutions in many countries.

\section{Botulinum-A toxin injection into the detrusor muscle}

Botulinum neurotoxin type A, synthesised in Clostridium botulinum, is the disease agent for botulism and the most potent natural poison. From the structural point of view the toxin is a $150 \mathrm{kDa}$ amino acid di-chain molecule consisting of a light $(50 \mathrm{kDa})$ and a heavy chain $(100 \mathrm{kDa})$ that are linked by a disulphide bond. ${ }^{47}$ The toxicity of the botulinum toxin is a result of a multistep mechanism. ${ }^{48}$ The neurotoxin binds to the presynaptic nerve endings of cholinergic neurons and enters the neuron by receptor-mediated endocytosis. There the catalytic domain specifically cleaves the SNAP-25 protein essential for normal synaptic vesical fusion. This cleavage results in the inhibition of neuronal acetylcholine secretion, ultimately leading to a temporary chemodenervation and the loss or reduction of neuronal activity at the target organs. ${ }^{47,49}$ In general, this chemodenervation is fully reversible. Regeneration process relies on the formation of functional neuronal sprouts that reconnect presynaptic nerve endings with their target organs (muscles or glands). ${ }^{50}$ After the blocked presynaptic nerve ending re-established the connections to the target organ, the sprouts have been shown to disappear. ${ }^{50}$

The underlying hypothesis for the use of the neurotoxin for bladder disorders was based on the study of Dickson and Shevky ${ }^{51}$ suggesting that parasympathetic action may be blocked by botulinum-A toxin. ${ }^{51}$ Disorders of the parasympathetic autonomic nervous system such as achalasia and hyperhidrosis have been successfully treated with botulinum-A toxin injections. $^{52-54} \mathrm{~A}$ marked loss of contraction in a rat bladder after acute botulinum poisoning with decrease in acetylcholine release at motor nerve stimulation was observed by Carpenter. ${ }^{55}$

The effect of injecting botulinum-A toxin into the human detrusor muscle in patients with neurogenic detrusor overactivity was first reported in 2000 in a nonrandomised prospective study. ${ }^{56,57}$ The patients with spinal cord injury selected for a preliminary study had severe neurogenic detrusor overactivity and suffered from incontinence resistant to anticholinergic drugs. ${ }^{56}$ They emptied their bladder by intermittent self-catheterisation. Patients with low bladder compliance due to organic detrusor muscle changes or fibrosis were excluded. In total, 200-400 U of botulinum-A toxin (Botox ${ }^{\circledR}$ ) were injected into the detrusor muscle sparing the trigone. A total of 19 patients were regularly observed over a period of 9 months by clinical and urodynamic checks. A 6 weeks follow-up after injections showed a significant increase in the reflex volume and in the maximum cystometric bladder capacity. There was also a significant decrease in the maximum detrusor voiding pressure. At the 36 weeks follow-up, ongoing improvement occurred. The amount of anticholinergics could be reduced or even completely abolished. Continence was restored in all but two patients and the patients' satisfaction was high. The recent experience increased to approximately 200 patients with the same results and profile. ${ }^{58}$

In 2002, the efficacy of botulinum-A toxin in children with neurogenic detrusor overactivity due to meningomyelocele was described. ${ }^{59}$ The 17 children were using clean intermittent catheterisation and anticholinergic drugs. Included were children aged 1-16 years with either neurogenic detrusor overactivity or high intravesical pressure exceeding $40 \mathrm{~cm} \mathrm{H}_{2} \mathrm{O}$ resistant to anticholinergic medication or presenting with unacceptable side effects. In all, 85-300 U of botulinum-A toxin (Botox ${ }^{\circledR}$ ) were injected into the detrusor muscle and urodynamic checks were performed 2-4 weeks after injection. The mean reflex volume, mean maximum bladder capacity and mean detrusor compliance increased, and the mean maximal detrusor pressure decreased. All results were significant and continence could be restored until at least the 4 weeks follow-up. In a recent study from the same group, the follow-up has been extended to 6 months, suggesting that in children also the botulinum-A toxin injection into the detrusor muscle is effective for a period of about 6 months, and then reinjection is necessary. ${ }^{60}$ The results of these nonrandomised prospective studies are promising, especially considering the fact that in both studies the patients included were difficult cases for conservative treatment. In summary, at present botulinum-A toxin injections into the detrusor muscle seem to be indicated in spinal cord injured patients with incontinence due to neurogenic detrusor overactivity. This treatment option seems to establish its indication in cases where anticholinergic medication fails or is intolerable and appears to be a valuable alternative to surgery. However, randomised and placebo-controlled studies are absolute necessary to prove the effect on an evidence-based foundation, to determine the exact duration of the effect and to optimise the injection technique concerning toxin dilution and number of injection sites.

\section{Conclusion}

Intravesical treatment strategies in patients with neurogenic detrusor overactivity may provide alternatives to established therapies such as oral anticholinergics. The selectivity of the intravesical treatment and the reduction or even the absence of side effects are major advantages of this topical approach. The intravesical instillation of resiniferatoxin and the injection of botulinum-A toxin into the detrusor muscle are promising new options; however, randomised placebo-controlled studies to prove their safety and efficacy are still missing. 


\section{References}

1 Abrams $\mathrm{P}$ et al. The standardisation of terminology of lower urinary tract function: report from the Standardisation Sub-committee of the International Continence Society. Neurourol Urodyn 2002; 21: 167-178.

2 de Groat WC et al. Organization of the sacral parasympathetic reflex pathways to the urinary bladder and large intestine. J Auton Nerv Syst 1981; 3: 135-160.

3 Reuther K, Aagaard J, Jensen KS. Lignocaine test and detrusor instability. Br J Urol 1983; 55: 493-494.

4 Higson RH, Smith JC, Hills W. Intravesical lignocaine and detrusor instability. Br J Urol 1979; 51: 500-503.

5 Lapointe SP, Wang B, Kennedy WA, Shortliffe LM. The effects of intravesical lidocaine on bladder dynamics of children with myelomeningocele. J Urol 2001; 165 (6 Part 2): 2380-2382.

6 McInerney PD, Grant A, Chawla J, Stephenson TP. The effect of intravesical Marcain instillation on hyperreflexic detrusor contractions. Paraplegia 1992; 30: 127-130.

7 Yokoyama $\mathrm{O}$ et al. Diagnostic value of intravesical lidocaine for overactive bladder. J Urol 2000; 164: 340-343.

8 Yokoyama $\mathrm{O}$ et al. Urodynamic effects of intravesical instillation of lidocaine in patients with overactive detrusor. J Urol 1997; 157: 1826-1830.

9 Avelino A, Cruz F. Peptide immunoreactivity and ultrastructure of rat urinary bladder nerve fibers after topical desensitization by capsaicin or resiniferatoxin. Auton Neurosci 2000; 86: 37-46.

10 Yiangou Y et al. Capsaicin receptor VR1 and ATP-gated ion channel P2X3 in human urinary bladder. BJU Int 2001; 87: 774-779.

11 Fowler CJ et al. Intravesical capsaicin for neurogenic bladder dysfunction. Lancet 1992; 339: 1239.

12 Wiart L et al. The effects of capsaicin on the neurogenic hyperreflexic detrusor. A double blind placebo controlled study in patients with spinal cord disease. Preliminary results. Spinal Cord 1998; 36: 95-99.

13 de Seze $\mathbf{M}$ et al. Capsaicin and neurogenic detrusor hyperreflexia: a double-blind placebo-controlled study in 20 patients with spinal cord lesions. Neurourol Urodyn 1998; 17: 513-523.

14 Petersen T, Nielsen JB, Schroder HD. Intravesical capsaicin in patients with detrusor hyper-reflexia - a placebocontrolled cross-over study. Scand J Urol Nephrol 1999; 33: 104-110.

15 de Seze $M$ et al. Intravesical instillation of capsaicin in urology: a review of the literature. Eur Urol 1999; 36: 267-277.

16 Avelino A, Cruz F, Coimbra A. Lidocaine prevents noxious excitation of bladder afferents induced by intravesical capsaicin without interfering with the ensuing sensory desensitization: an experimental study in the rat. $J$ Urol 1998; 159: 567-570.

17 Fowler CJ. Intravesical treatment of overactive bladder. Urology 2000; 55 (5A Suppl): 60-64; discussion 66.

18 Cruz F et al. Desensitization of bladder sensory fibers by intravesical capsaicin has long lasting clinical and urodynamic effects in patients with hyperactive or hypersensitive bladder dysfunction. J Urol 1997; 157: 585-589.

19 De Ridder D et al. Intravesical capsaicin as a treatment for refractory detrusor hyperreflexia: a dual center study with long-term followup. J Urol 1997; 158: 2087-2092.

20 Yoshimura N, Chancellor MB. Current and future pharmacological treatment for overactive bladder. $J$ Urol 2002; 168: 1897-1913.
21 Cruz F, Guimaraes M, Silva C, Reis M. Suppression of bladder hyperreflexia by intravesical resiniferatoxin. Lancet 1997; 350: 640-641.

22 Giannantoni A et al. Intravesical capsaicin versus resiniferatoxin in patients with detrusor hyperreflexia: a prospective randomized study. J Urol 2002; 167: 1710-1714.

23 Lazzeri $\mathrm{M}$ et al. Intravesical resiniferatoxin for the treatment of detrusor hyperreflexia refractory to capsaicin in patients with chronic spinal cord diseases. Scand J Urol Nephrol 1998; 32: 331-334.

24 Silva C, Rio ME, Cruz F. Desensitization of bladder sensory fibers by intravesical resiniferatoxin, a capsaicin analog: long-term results for the treatment of detrusor hyperreflexia. Eur Urol 2000; 38: 444-452.

25 Lazzeri $\mathrm{M}$ et al. Intravesical resiniferatoxin for the treatment of hypersensitive disorder: a randomized placebo controlled study. J Urol 2000; 164 (3 Part 1): 676-679.

26 Reinscheid RK et al. Orphanin FQ: a neuropeptide that activates an opioidlike $\mathrm{G}$ protein-coupled receptor. Science 1995; 270: 792-794.

27 Meunier JC et al. Isolation and structure of the endogenous agonist of opioid receptor-like ORL1 receptor. Nature 1995; 377: 532-535.

28 Lecci A, Giuliani S, Meini S, Maggi CA. Nociceptin and the micturition reflex. Peptides 2000; 21: 1007-1021.

29 Lazzeri $\mathrm{M}$ et al. Urodynamic and clinical evidence of acute inhibitory effects of intravesical nociceptin/orphanin FQ on detrusor overactivity in humans: a pilot study. $J$ Urol 2001; 166: 2237-2240.

30 Lazzeri $\mathrm{M}$ et al. Urodynamic effects of intravesical nociceptin/orphanin FQ in neurogenic detrusor overactivity: a randomized, placebo-controlled, double-blind study. Urology 2003; 61: 946-950.

31 Glickman S, Tsokkos N, Shah PJ. Intravesical atropine and suppression of detrusor hyperconrractility in the neuropathic bladder. A preliminary study. Paraplegia 1995; 33: 36-39.

32 Deaney C, Glickman S, Gluck T, Malone-Lee JG. Intravesical atropine suppression of detrusor hyperreflexia in multiple sclerosis. J Neurol Neurosurg Psychiatry 1998; 65: 957-958.

33 Enskat R, Deaney CN, Glickman S. Systemic effects of intravesical atropine sulphate. BJU Int 2001; 87: 613-616.

34 Brendler CB, Radebaugh LC, Mohler JL. Topical oxybutynin chloride for relaxation of dysfunctional bladders. J Urol 1989; 141: 1350-1352.

35 Madersbacher H, Jilg G. Control of detrusor hyperreflexia by the intravesical instillation of oxybutynine hydrochloride. Paraplegia 1991; 29: 84-90.

36 Massad CA, Kogan BA, Trigo-Rocha FE. The pharmacokinetics of intravesical and oral oxybutynin chloride. J Urol 1992; 148 (2 (Part 2)): 595-597.

37 Madersbacher $\mathrm{H}$, Knoll $\mathrm{M}$. Intravesical application of oxybutynine: mode of action in controlling detrusor hyperreflexia. Preliminary results. Eur Urol 1995; 28: 340-344.

38 Lehtoranta K et al. Pharmacokinetics, efficacy, and safety of intravesical formulation of oxybutynin in patients with detrusor overactivity. Scand J Urol Nephrol 2002; 36: 18-24.

39 Buyse $\mathrm{G}$ et al. Intravesical oxybutynin for neurogenic bladder dysfunction: less systemic side effects due to reduced first pass metabolism. J Urol 1998; 160 (3 (Part 1)): 892-896. 
40 De Wachter S, Wyndaele JJ. Intravesical oxybutynin: a local anesthetic effect on bladder C afferents. J Urol 2003; 169: 1892-1895.

41 Greenfield SP, Fera M. The use of intravesical oxybutynin chloride in children with neurogenic bladder. J Urol 1991; 146 (2 (Part 2)): 532-534.

42 Amark $\mathrm{P}$ et al. Pharmacokinetics and effects of intravesical oxybutynin on the paediatric neurogenic bladder. Br J Urol 1998; 82: 859-864.

43 Amark P, Bussman G, Eksborg S. Follow-up of long-time treatment with intravesical oxybutynin for neurogenic bladder in children. Eur Urol 1998; 34: 148-153.

44 Kasabian NG et al. The use of intravesical oxybutynin chloride in patients with detrusor hypertonicity and detrusor hyperreflexia. J Urol 1994; 151: 944-945.

45 Palmer LS, Zebold K, Firlit CF, Kaplan WE. Complications of intravesical oxybutynin chloride therapy in the pediatric myelomeningocele population. J Urol 1997; 157: 638-640.

46 Pannek J, Sommerfeld HJ, Botel U, Senge T. Combined intravesical and oral oxybutynin chloride in adult patients with spinal cord injury. Urology 2000; 55: 358-362.

47 Montecucco C, Schiavo G. Structure and function of tetanus and botulinum neurotoxins. $Q$ Rev Biophys 1995; 28: $423-472$.

48 Simpson LL. Kinetic studies on the interaction between botulinum toxin type A and the cholinergic neuromuscular junction. J Pharmacol Exp Ther 1980; 212: 16-21.

49 Lacy DB et al. Crystal structure of botulinum neurotoxin type A and implications for toxicity. Nat Struct Biol 1998; 5: 898-902.

50 de Paiva A et al. Functional repair of motor endplates after botulinum neurotoxin type A poisoning: biphasic switch of synaptic activity between nerve sprouts and their parent terminals. Proc Natl Acad Sci USA 1999; 96: 3200-3205.
51 Dickson EC, Shevky R. Studies on manner in which the toxin of clostridium botulinum acts upon the body. I. The effect upon the autonomic nervous system. $J$ Exp Med 1923; 37: 711-731.

52 Annese $\mathrm{V}$ et al. Intrasphincteric injection of botulinum toxin is effective in long-term treatment of esophageal achalasia. Muscle Nerve 1998; 21: 1540-1542.

53 Naumann $\mathrm{M}$ et al. Botulinum toxin for palmar hyperhidrosis. Lancet 1997; 349: 252.

54 Naumann M, Zellner M, Toyka KV, Reiners K. Treatment of gustatory sweating with botulinum toxin. Ann Neurol 1997; 42: 973-975.

55 Carpenter FG. Motor responses of the response of the urinary bladder and skeletal muscle in botulinum toxin intoxicated rats. J Physiol 1967; 1988: 1-11.

56 Schurch B et al. Botulinum-A toxin for treating detrusor hyperreflexia in spinal cord injured patients: a new alternative to anticholinergic drugs?. Preliminary results. J Urol 2000; 164 (3 (Part 1)): 692-697.

57 Schurch B, Schmid DM, Stohrer M. Treatment of neurogenic incontinence with botulinum toxin A. $N$ Engl $J$ Med 2000; 342: 665.

58 Reitz A et al. European experience of 184 cases treated with botulinum-A toxin injections into the detrusor muscle for neurogenic incontinence. Neurourol Urodyn 2002; 21: 427-428.

59 Schulte-Baukloh $\mathrm{H}$ et al. Efficacy of botulinum-a toxin in children with detrusor hyperreflexia due to myelomeningocele: preliminary results. Urology 2002; 59: 325-327; discussion 327-328.

60 Schulte-Baukloh H, Michael T, Sturzebecher B, Knispel HH. Botulinum-a toxin detrusor injection as a novel approach in the treatment of bladder spasticity in children with neurogenic bladder. Eur Urol 2003; 44: 139-143. 